\title{
A LARGE-AREA SURVEY FOR RADIO PULSARS AT HIGH GALACTIC LATITUDES
}

\author{
B. A. JaCOBY ${ }^{1,2}$, M. BAILES ${ }^{3}$, S. M. ORD ${ }^{3,4}$, R. T. EdwARdS ${ }^{5}$, And S. R. KulKarni ${ }^{1}$ \\ ${ }^{1}$ Department of Astronomy, California Institute of Technology, MS 249-17, Pasadena, CA 91125, USA; baj@ astro.caltech.edu, srk@astro.caltech.edu \\ 2 Naval Research Laboratory, Code 7213, 4555 Overlook Avenue, SW, Washington, DC 20375, USA \\ ${ }^{3}$ Centre for Astrophysics and Supercomputing, Swinburne University of Technology, P.O. Box 218, Hawthorn, VIC 31122, Australia; mbailes@ swin.edu.au \\ ${ }^{4}$ School of Physics, University of Sydney, A28, NSW 2006, Australia; ord@ physics.usyd.edu.au \\ ${ }^{5}$ Australia Telescope National Facility, CSIRO, P.O. Box 76, Epping, NSW 1710, Australia; Russell.Edwards@csiro.au \\ Received 2007 February 19; accepted 2009 May 12; published 2009 June 26
}

\begin{abstract}
We have completed a survey for pulsars at high Galactic latitudes with the $64 \mathrm{~m}$ Parkes radio telescope. Observing with the 13 beam multibeam receiver at a frequency of $1374 \mathrm{MHz}$, we covered 4150 square degrees in the region $-100^{\circ} \leqslant l \leqslant 50^{\circ}, 15^{\circ} \leqslant|b| \leqslant 30^{\circ}$ with 7232 pointings of $265 \mathrm{~s}$ each, thus extending the Swinburne Intermediate Latitude Pulsar Survey a further $15^{\circ}$ on either side of the Galactic plane. The signal from each beam was processed by a 96 channel $\times 3 \mathrm{MHz} \times 2$ polarization filterbank, with the detected power in the two polarizations of each frequency channel summed and digitized with 1 bit sampling every $125 \mu \mathrm{s}$, giving good sensitivity to millisecond pulsars with low or moderate dispersion measure. The resulting 2.4 TB data set was processed using standard pulsar search techniques with the workstation cluster at the Swinburne Centre for Astrophysics and Supercomputing. This survey resulted in the discovery of 26 new pulsars including seven binary and/or millisecond pulsars, and redetected 36 previously known pulsars. We describe the survey methodology and results, and present timing solutions for the 19 newly discovered slow pulsars, as well as for nine slow pulsars discovered the Swinburne Intermediate Latitude Pulsar Survey that had no previous timing solutions. Even with a small sampling interval, $1374 \mathrm{MHz}$ center frequency, and a large mid-latitude survey volume we failed to detect any very rapidly spinning pulsars. Evidently, such "submillisecond" pulsars are rare.
\end{abstract}

Key words: pulsars: general - stars: neutron - surveys

\section{INTRODUCTION}

Since the discovery of the first radio pulsar (Hewish et al. 1968), a great deal of effort in radio astronomy has been expended searching for pulsars, with about 1800 pulsars known today. Until the late 1990s, most pulsars surveys focused on the region of sky near the plane of our galaxy since that is where the density of pulsars is highest. Also, early surveys usually were conducted at frequencies around $400 \mathrm{MHz}$ because of pulsars' steep radio spectra, and because the larger beam produced by a given telescope at lower frequencies allowed for more rapid coverage of the sky, or conversely, longer integration on a given point in the sky, given a region of sky to cover and a fixed allocation of observing time.

However, there are clear advantages to searching for pulsars at higher frequencies. Most notably, dispersion and interstellar scattering are mitigated, allowing for better time resolution and hence sensitivity to pulsars with large dispersion measures (DMs) and narrow pulses. Also, the galactic synchrotron background has a steep spectrum and contributes substantially less to the total system temperature at higher frequencies. The main disadvantage of high-frequency surveys, namely the slow sky coverage resulting from a small telescope beam, has been mitigated by the innovative 13 beam multibeam receiver package at the Parkes radio telescope (Staveley-Smith et al. 1996) and 13 accompanying analog filterbanks which provide a combined beam area on the sky about 25\% larger than the $70 \mathrm{~cm}$ system used in the Parkes Southern Pulsar Survey (Manchester et al. 1996). The Parkes Multibeam Pulsar Survey covering 1500 square degrees within $5^{\circ}$ of the Galactic plane has used this system to great effect, roughly doubling the number of pulsars known before this survey began (Manchester et al. 2001; Lorimer et al. 2006).
While pulsars descend from short-lived massive stars which are born and die in the Galactic disk, older pulsars have had time to migrate away from the disk and will be found at moderate to high galactic latitudes. Millisecond pulsars (MSPs) which spin roughly a hundred times or more each second are in this older group, having lived a life as a "normal" pulsar, then later being "recycled" to very fast rotation rates by accreting matter from an evolved binary companion. It is these objects which promise to reveal the neutron star equation of state: discovering ever faster spinning pulsars constrains the size of neutron stars, and measuring effects predicted by Einstein's general relativity in binary systems allows us to determine their masses. Although there are fewer pulsars per unit solid angle at high Galactic latitudes than in the plane of the galaxy, a shallow survey of this relatively neglected part of the sky provides an efficient means for discovering recycled pulsars, as demonstrated by the Swinburne Intermediate Latitude Pulsar Survey in a region between $5^{\circ}$ and $15^{\circ}$ from the Galactic plane (Edwards \& Bailes 2001; Edwards et al. 2001). Here, we describe the results of a $21-\mathrm{cm}$ multibeam survey for pulsars at higher Galactic latitudes, between $15^{\circ}$ and $30^{\circ}$ from the Galactic plane. For a more complete description of this survey, see Jacoby (2005).

\section{OBSERVATIONS AND ANALYSIS}

Between 2001 January and 2002 December, we observed roughly 4150 square degrees in the region $-100^{\circ} \leqslant l \leqslant 50^{\circ}$, $15^{\circ} \leqslant|b| \leqslant 30^{\circ}$ in 7232 individual pointings of $265 \mathrm{~s}$ with the 64-m Parkes radio telescope at a central frequency of $1374 \mathrm{MHz}$. Using the 13-beam multibeam receiver system, the signal from each beam was channelized by a 96 channel $\times 3 \mathrm{MHz} \times$ 2 polarization analog filterbank; the powers from each polarization pair were summed, integrated, and 1 bit sampled at $125 \mu \mathrm{s}$ 


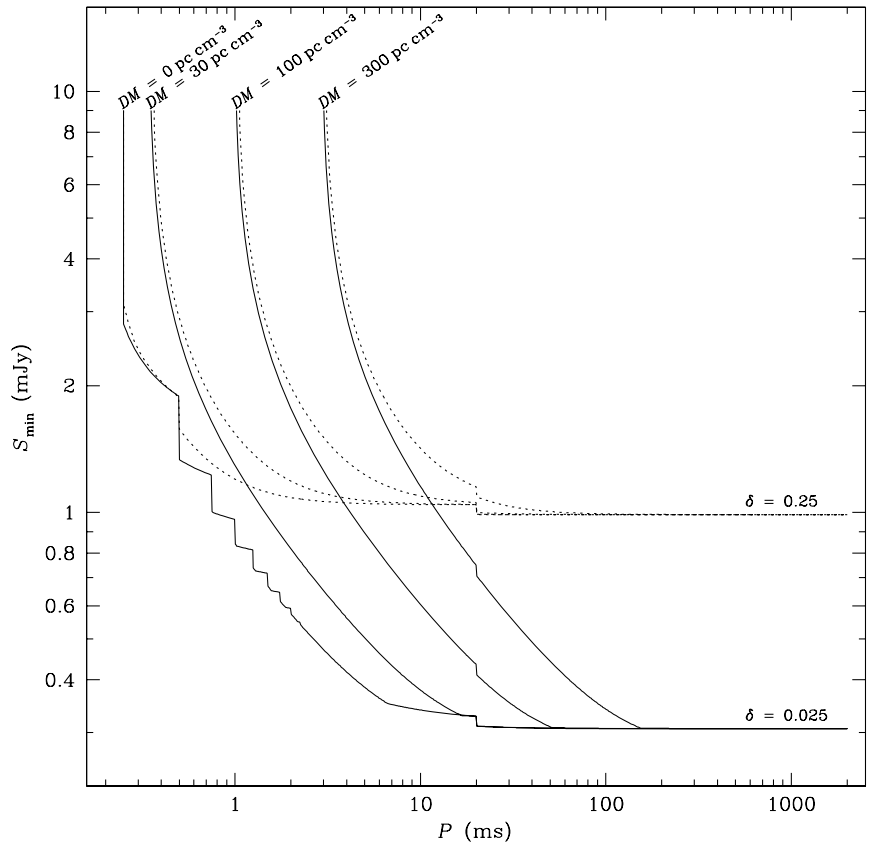

Figure 1. Survey sensitivity. Curves show minimum detectable $1400 \mathrm{MHz}$ flux density as a function of spin period for pulsars with large (the dotted curves) and small (the solid curves) intrinsic duty cycles $(\delta)$ for a range of dispersion measures as labeled.

intervals. The resulting 94,016 survey beams comprising $2.4 \mathrm{~TB}$ in total were written to 98 DLT tapes for later analysis. The observing and data processing procedures were virtually identical to those described by Edwards et al. (2001). Our observing hardware and methodology were identical to the Parkes Multibeam Pulsar Survey, except that our integration time per pointing was shorter (265 s compared to $2100 \mathrm{~s}$ ) and our sampling interval was half as long (125 $\mu$ s compared to $250 \mu \mathrm{s}$ ). The Parkes High Latitude Pulsar Survey, covering the region $220^{\circ}<l<260^{\circ}$, $|b|<60^{\circ}$, also employed the shorter $125 \mu$ s sampling interval and $265 \mathrm{~s}$ integration time (Burgay et al. 2006).

The minimum detectable flux density $S_{\min }$ for a radio periodicity search can be calculated by a modified form of the radiometer equation:

$$
S_{\min }=\frac{\alpha \beta\left(T_{\text {rec }}+T_{\text {sky }}\right)}{G\left(2 \Delta v t_{\text {int }}\right)^{1 / 2}}\left(\frac{\delta}{1-\delta}\right)^{1 / 2},
$$

where $\alpha$ is the threshold signal-to-noise ratio $(\mathrm{S} / \mathrm{N}), \beta \approx 1.5$ is an efficiency factor taking account of losses such as quantization error, $G$ is the telescope gain, $\Delta v$ is the observed bandwidth, $t_{\text {int }}$ is the integration time, $T_{\text {rec }}$ and $T_{\text {sky }}$ are the receiver and sky contributions to the system noise temperature, and $\delta$ is the effective fractional duty cycle of the periodic signal, including contributions from the post-detection integration, dispersion smearing, finite DM search step size, and scattering. Figure 1 shows representative sensitivity curves for this survey. In our calculations, we have assumed that the pulsar signal contains $(2 \delta)^{-1}$ equally significant harmonics in the frequency domain.

Our large data set was analyzed with the cluster of 64 Compaq Alpha workstations at Swinburne University of Technology's Centre for Astrophysics and Supercomputing. The filterbank data from each survey beam were padded with 32 empty channels with appropriate frequency spacing so that dispersion was a linear function of channel number in this 128 channel space. First, each channel was searched for strong, narrow- band radio-frequency interference (RFI), and affected channels were masked. We then used the "tree" dedispersion algorithm (Taylor 1974) to break the 128 channels into 16 sub-bands, each dedispersed at a range of different DMs. This sub-band data could then be efficiently dedispersed and summed to form one of 374 trial DMs up to a maximum $562.5 \mathrm{pc} \mathrm{cm}^{-3}$. Once the sub-band DM reached twice the diagonal DM of $17 \mathrm{pc} \mathrm{cm}^{-3}$ (when roughly one sample period of smearing occurs within an individual channel), adjacent time samples were summed (thereby doubling the diagonal DM) and the process repeated until the maximum search DM was reached.

The periodicity search used an adaptation of the search software from the Parkes Southern Pulsar Survey (Manchester et al. 1996) and followed standard pulsar search procedures. Briefly, the Fourier transform was computed for a given trial DM and searched for harmonically related patterns with a fundamental frequency greater than $1 / 12 \mathrm{~Hz}$. The large list of possible candidates in the three-dimensional space of repetition frequency, number of harmonics summed, and DM was consolidated into a list of the best 99 suspects from each survey beam. Each of these was then subjected to a time-domain optimization in spin period $(P)$ and DM. For each beam, the 48 most promising suspects from this optimization were saved for further scrutiny. We note that, because the maximum number of harmonics summed was 16 , our search sensitivity was not optimal for pulsars with very small duty cycles $(\lesssim 3 \%)$.

Candidate pulsars were selected from the search suspects using a variety of fixed and adaptive filters, and finally, by human examination. Typically, the roughly 45,000 suspects from a given tape were considered together. Filters were constructed to eliminate known interference fluctuation frequencies and to enforce criteria to eliminate other low-quality suspects such as short-period suspects with large relative DM error. The S/N threshold was set at 9 for suspects with $P \geqslant 20 \mathrm{~ms}$ and 9.5 for shorter-period suspects to help eliminate the many spurious short-period signals found in our data. Finally, adaptive filters were used to eliminate signals that appeared at nearly the same period separated by many telescope beam widths many times on one tape. These automated parameter-based filters and screens allowed us to cull the suspect list to fewer than 10,000 , each of which was then given much more human attention than would have been possible for the full set of suspects. Information considered in the human examination of selected suspects included graphical representations of optimized period and DM relative to the values found in the frequency domain search, $\mathrm{S} / \mathrm{N}$ as a function of $\mathrm{DM}$ trial, folded pulse profile in each of several frequency sub-bands, best integrated pulse profile, and folded pulse profile in each of several time sub-integrations. Plausible pulsar candidates were reobserved, with the observation time depending on the strength of the candidate.

\section{DETECTED PULSARS}

In addition to seven recycled pulsars (Jacoby et al. 2003; Jacoby et al. 2007; B. A. Jacoby et al. 2009, in preparation), this survey discovered 19 new slow pulsars. After confirmation, we began a roughly monthly timing program to determine phase-connected timing solutions for all slow pulsars using the Parkes 512 channel $\times 0.5 \mathrm{MHz} \times 2$ polarization filterbank in conjunction with the center beam of the multibeam receiver centered on $1390 \mathrm{MHz}$. Following standard pulse-timing procedures, folded profiles were cross-correlated with a template profile to determine times of arrival (TOAs). We used the standard 
Table 1

Timing Model Parameters for 19 New Slow Pulsars

\begin{tabular}{|c|c|c|c|c|c|c|}
\hline \multirow[t]{2}{*}{ Pulsar } & \multicolumn{6}{|c|}{ Parameter $^{\mathrm{a}}$} \\
\hline & $\alpha(\mathrm{J} 2000)$ & $\delta(\mathrm{J} 2000)$ & $\begin{array}{l}P \\
\text { (s) }\end{array}$ & $\begin{array}{c}P \text { Epoch } \\
\text { (MJD) }\end{array}$ & $\begin{array}{c}\dot{P} \\
\left(10^{-15}\right)\end{array}$ & $\begin{array}{c}\mathrm{DM}^{\mathrm{b}} \\
\left(\mathrm{pc} \mathrm{cm}^{-3}\right)\end{array}$ \\
\hline J0656-5449 & $06^{\mathrm{h}} 56^{\mathrm{m}} 48^{\mathrm{s}} .990(7)$ & $-54^{\circ} 49^{\prime} 14^{\prime \prime} 92(4)$ & $0.183156898795(2)$ & 53000.0 & $0.03191(9)$ & $67.5(10)$ \\
\hline J0709-5923 & $07^{\mathrm{h}} 09^{\mathrm{m}} 32^{\mathrm{s}} .533(8)$ & $-59^{\circ} 23^{\prime} 55^{\prime \prime} 60(4)$ & $0.485268383925(5)$ & 53000.0 & $0.1260(2)$ & $65(2)$ \\
\hline J1231-4609 & $12^{\mathrm{h}} 31^{\mathrm{m}} 45^{\mathrm{s}} \cdot 76(14)$ & $-46^{\circ} 09^{\prime} 45^{\prime \prime} \cdot 2(3)$ & $0.87723907778(4)$ & 53000.0 & $0.0380(17)$ & $76(7)$ \\
\hline $\mathrm{J} 1308-4650$ & $13^{\mathrm{h}} 18^{\mathrm{m}} 44^{\mathrm{s}} .589(19)$ & $-46^{\circ} 50^{\prime} 29^{\prime} .7(4)$ & $1.05883304424(3)$ & 53000.0 & $0.5259(16)$ & $66(10)$ \\
\hline J1333-4449 & $13^{\mathrm{h}} 33^{\mathrm{m}} 44^{\mathrm{s}} .829(5)$ & $-44^{\circ} 49^{\prime} 26^{\prime \prime} .22(10)$ & $0.345602948594(3)$ & 53000.0 & $0.00054(19)$ & 44.3(17) \\
\hline J1339-4712 & $13^{\mathrm{h}} 39^{\mathrm{m}} 56^{\mathrm{s}} .5886(18)$ & $-47^{\circ} 12^{\prime} 05^{\prime \prime} .52(3)$ & $0.1370546579332(4)$ & 53000.0 & $0.00053(2)$ & $39.9(6)$ \\
\hline $\mathrm{J} 1427-4158$ & $14^{\mathrm{h}} 27^{\mathrm{m}} 50^{\mathrm{s}} .770(9)$ & $-41^{\circ} 58^{\prime} 56^{\prime \prime} 3(3)$ & $0.586485556229(18)$ & 53000.0 & $0.6212(7)$ & $71(3))$ \\
\hline $\mathrm{J} 1536-3602$ & $15^{\mathrm{h}} 36^{\mathrm{m}} 17^{\mathrm{s}} .382(14)$ & $-36^{\circ} 02^{\prime} 58^{\prime \prime} .8(5)$ & $1.31975904174(5)$ & 53000.0 & $0.7900(19)$ & $96(6)$ \\
\hline J1609-1930 & $16^{\mathrm{h}} 09^{\mathrm{m}} 05^{\mathrm{s}} \cdot 35(12)$ & $-19^{\circ} 30^{\prime} 08(9)^{\prime \prime}$ & $1.55791724762(7)$ & 53000.0 & $0.509(3)$ & $37(7)$ \\
\hline J1612-2408 & $16^{\mathrm{h}} 12^{\mathrm{m}} 26^{\mathrm{s}} .06(3)$ & $-24^{\circ} 08^{\prime} 04(2)^{\prime \prime}$ & $0.92383371069(3)$ & 53000.0 & $1.5736(12)$ & $49(4)$ \\
\hline J1635-1511 & $16^{\mathrm{h}} 35^{\mathrm{m}} 47^{\mathrm{s}} \cdot 36(4)$ & $-15^{\circ} 11^{\prime} 52(3)^{\prime \prime}$ & $1.17938703902(8)$ & 53000.0 & $0.232(4)$ & $54(8)$ \\
\hline J1651-7642 & $16^{\mathrm{h}} 51^{\mathrm{m}} 07^{\mathrm{s}} .87(16)$ & $-76^{\circ} 42^{\prime} 39^{\prime \prime} .5(7)$ & $1.75531107981(18)$ & 53000.0 & $1.363(8)$ & $80(10)$ \\
\hline $\mathrm{J} 1652-1400$ & $16^{\mathrm{h}} 52^{\mathrm{m}} 16^{\mathrm{s}} .677(7)$ & $-14^{\circ} 00^{\prime} 27^{\prime \prime} .4(4)$ & $0.305447058241(3)$ & 53000.0 & $0.01758(15)$ & $49.5(13)$ \\
\hline J1714-1054 & $17^{\mathrm{h}} 14^{\mathrm{m}} 40^{\mathrm{s}} .122(5)$ & $-10^{\circ} 54^{\prime} 10^{\prime} \cdot 9(3)$ & $0.696278743075(9)$ & 53000.0 & $0.0588(4)$ & $51(3)$ \\
\hline $\mathrm{J} 1739+0612$ & $17^{\mathrm{h}} 39^{\mathrm{m}} 17^{\mathrm{s}} .966(4)$ & $+06^{\circ} 12^{\prime} 28^{\prime \prime} 4(10)$ & $0.234169035616(3)$ & 53000.0 & $0.15640(12)$ & $101.5(13)$ \\
\hline J1816-5643 & $18^{\mathrm{h}} 16^{\mathrm{m}} 36^{\mathrm{s}} \cdot 464(7)$ & $-56^{\circ} 43^{\prime} 42^{\prime \prime} .10(6)$ & $0.2179228818474(13)$ & 53000.0 & $0.00193(6)$ & $52.4(11)$ \\
\hline J1841-7845 & $18^{\mathrm{h}} 41^{\mathrm{m}} 26^{\mathrm{s}} .7(2)$ & $-78^{\circ} 45^{\prime} 25(2)^{\prime \prime}$ & $0.35360252723(4)$ & 53000.0 & $0.400(7)$ & $41(2)$ \\
\hline $\mathrm{J} 1846-7403$ & $18^{\mathrm{h}} 46^{\mathrm{m}} 13^{\mathrm{s}} \cdot 78(17)$ & $-74^{\circ} 03^{\prime} 04(2)^{\prime \prime}$ & $4.8788385261(5)$ & 53000.0 & $6.06(10)$ & $97(20)$ \\
\hline J1946-1312 & $19^{\mathrm{h}} 46^{\mathrm{m}} 57^{\mathrm{s}} .829(10)$ & $-13^{\circ} 12^{\prime} 36^{\prime \prime} 4(6)$ & $0.491865489484(6)$ & 53000.0 & $1.9866(3)$ & $60(2)$ \\
\hline
\end{tabular}

Notes.

a Figures in parentheses are uncertainties in the last digit quoted. Uncertainties are calculated from twice the formal error produced by TEMPO.

${ }^{\mathrm{b}} \mathrm{DM}$ determined from discovery or confirmation observation across $288 \mathrm{MHz}$ wide observing band at $1374 \mathrm{MHz}$.

Table 2

Derived Parameters for 19 New Slow Pulsars

\begin{tabular}{|c|c|c|c|c|c|c|c|c|c|}
\hline \multirow[t]{2}{*}{ Pulsar } & \multicolumn{9}{|c|}{ Parameter } \\
\hline & $\mathrm{S} / \mathrm{N}^{\mathrm{a}}$ & $\begin{array}{l}w_{50} \\
(\mathrm{~ms})\end{array}$ & $\begin{array}{l}w_{10} \\
(\mathrm{~ms})\end{array}$ & $\begin{array}{c}l \\
(\operatorname{deg})\end{array}$ & $\begin{array}{c}b \\
(\mathrm{deg})\end{array}$ & $\begin{array}{c}d^{\mathrm{b}} \\
(\mathrm{kpc})\end{array}$ & $\begin{array}{c}|z| \\
(\mathrm{kpc})\end{array}$ & $\begin{array}{c}\tau_{c} \\
(\mathrm{Myr})\end{array}$ & $\begin{array}{c}B \\
\left(10^{12} \mathrm{G}\right)\end{array}$ \\
\hline J0656-5449 & 11.1 & 5.4 & 10.2 & 264.80 & -21.14 & 3.9 & 1.4 & 91 & 0.077 \\
\hline J0709-5923 & 14.7 & 5.1 & 11.5 & 270.03 & -20.90 & 3.3 & 1.2 & 61 & 0.25 \\
\hline $\mathrm{J} 1231-4609$ & 18.6 & 44.6 & 55.5 & 299.38 & +16.57 & 2.4 & 0.69 & 370 & 0.18 \\
\hline $\mathrm{J} 1308-4650$ & 12.5 & 54.6 & 69.9 & 306.01 & +15.93 & 1.9 & 0.53 & 32 & 0.76 \\
\hline J1333-4449 & 15.9 & 2.4 & 9.7 & 310.77 & +17.40 & 1.4 & 0.41 & 10,000 & 0.013 \\
\hline $\mathrm{J} 1339-4712$ & 21.9 & 2.5 & 4.9 & 311.42 & +14.87 & 1.2 & 0.31 & 4100 & 0.0086 \\
\hline J1427-4158 & 11.9 & 19.2 & 23.9 & 321.48 & +17.39 & 2.0 & 0.61 & 15 & 0.61 \\
\hline $\mathrm{J} 1536-3602$ & 57.7 & 84.6 & 98.0 & 336.55 & +15.84 & 3.0 & 0.82 & 26 & 1.0 \\
\hline J1609-1930 & 19.8 & 14.6 & 26.8 & 354.07 & +23.18 & 1.4 & 0.55 & 49 & 0.90 \\
\hline J1612-2408 & 16.9 & 20.8 & 31.8 & 351.01 & +19.45 & 1.6 & 0.54 & 9.3 & 1.2 \\
\hline $\mathrm{J} 1635-1511$ & 23.6 & 39.6 & 262.0 & 2.06 & +21.08 & 1.9 & 0.69 & 81 & 0.53 \\
\hline $\mathrm{J} 1651-7642$ & 21.9 & 83.6 & 104.9 & 315.15 & -19.95 & 2.9 & 0.97 & 20 & 1.6 \\
\hline $\mathrm{J} 1652-1400$ & 22.9 & 11.8 & 24.1 & 5.60 & +18.58 & 1.7 & 0.53 & 280 & 0.74 \\
\hline J1714-1054 & 24.7 & 7.7 & 77.2 & 11.49 & +15.78 & 1.6 & 0.44 & 190 & 0.20 \\
\hline $\mathrm{J} 1739+0612$ & 17.2 & 7.5 & 16.5 & 30.26 & +18.86 & $>17$ & $>5.6$ & 24 & 0.19 \\
\hline J1816-5643 & 12.3 & 6.6 & 22.6 & 337.67 & -17.90 & 1.5 & 0.46 & 1800 & 0.020 \\
\hline $\mathrm{J} 1841-7845$ & 17.6 & 22.8 & 41.4 & 315.46 & -26.08 & 1.4 & 0.63 & 14 & 0.38 \\
\hline J1846-7403 & 30.9 & 98.2 & 731.4 & 320.68 & -25.65 & $>12$ & $>5.2$ & 13 & 5.5 \\
\hline J1946-1312 & 11.9 & 11.6 & 18.9 & 27.08 & -18.18 & 2.2 & 0.69 & 3.9 & 1.0 \\
\hline
\end{tabular}

Notes.

${ }^{\text {a }}$ For pulsars detected in multiple survey beams, S/N of strongest detection.

b Distance estimated from dispersion measure using model of Cordes \& Lazio (2002).

pulsar-timing package TEMPO, ${ }^{6}$ along with the Jet Propulsion Laboratory's DE405 ephemeris for all timing analysis. TOA uncertainties for each pulsar were scaled to achieve reduced $\chi^{2} \simeq 1$ in order to improve the estimation of parameter uncertainties. Timing solutions for these pulsars are given in Table 1

$\overline{6 \text { http://www.atnf.csiro.au/research/pulsar/tempo/ }}$ and derived parameters are given in Table 2. Average pulse profiles are shown in Figure 2, and timing residuals relative to the models in Table 1 are shown in Figures 3 and 4.

The timing behavior of these objects is typical of slow pulsars. Three of these slow pulsars (J1333-4449, J1339-4712, and $\mathrm{J} 1816-5643)$ have relatively large characteristic ages $\left(\tau_{c}=\right.$ $\left.P / 2 \dot{P}>10^{9} \mathrm{yr}\right)$ and weak inferred surface dipole magnetic 


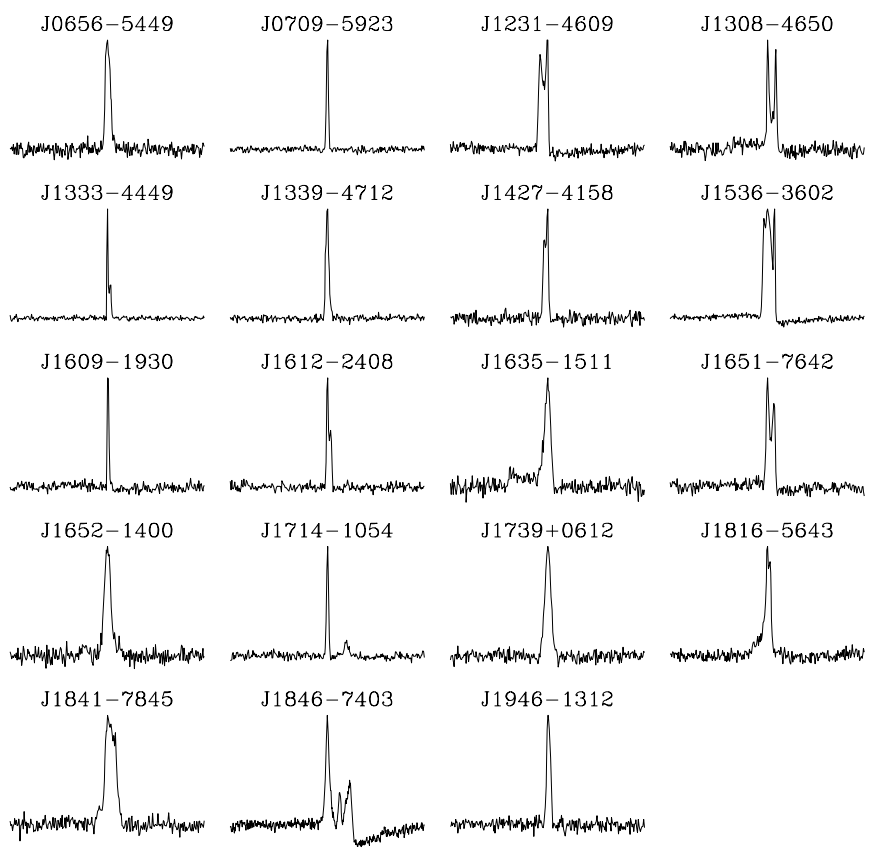

Figure 2. Pulse profiles of 19 new slow pulsars. The negative intensity seen in pulsars such as PSR J1846-7403 is an artifact of the filterbank digitizer's high-pass filter.
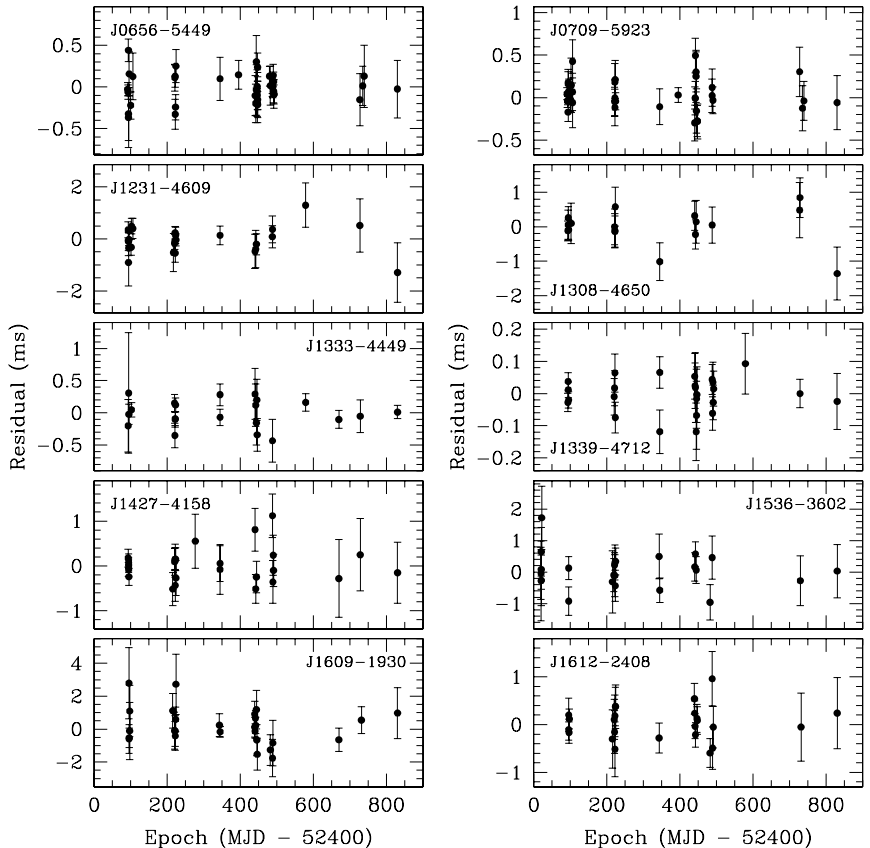

Figure 3. Timing residuals for 10 new slow pulsars.

fields $\left(B=3.2 \times 10^{19} \mathrm{Gs}^{-1 / 2} \sqrt{P \dot{P}} \approx 10^{10} \mathrm{G}\right.$ ) compared to other slow pulsars. In Figure 5, we plot $B$ versus $P$ for known pulsars with measured $\dot{P}$ and indicate the objects found in this survey. These three objects fall in the transition region in $B-P$ space between the predominantly isolated slow pulsars and the recycled pulsars found most often in binary systems.

In addition to the 26 pulsars discovered in this survey, 36 previously known pulsars were detected (Table 3 ). Of the 30 previously known pulsars we failed to detect (Table 4), only two have published flux densities at $1400 \mathrm{MHz}\left(S_{1400}\right)$ greater than $0.4 \mathrm{mJy}$. We note that in the case of one extremely convincing MSP candidate-later confirmed as PSR J1741+1351 (Jacoby et al. 2007)-it took four reobservations at four times the
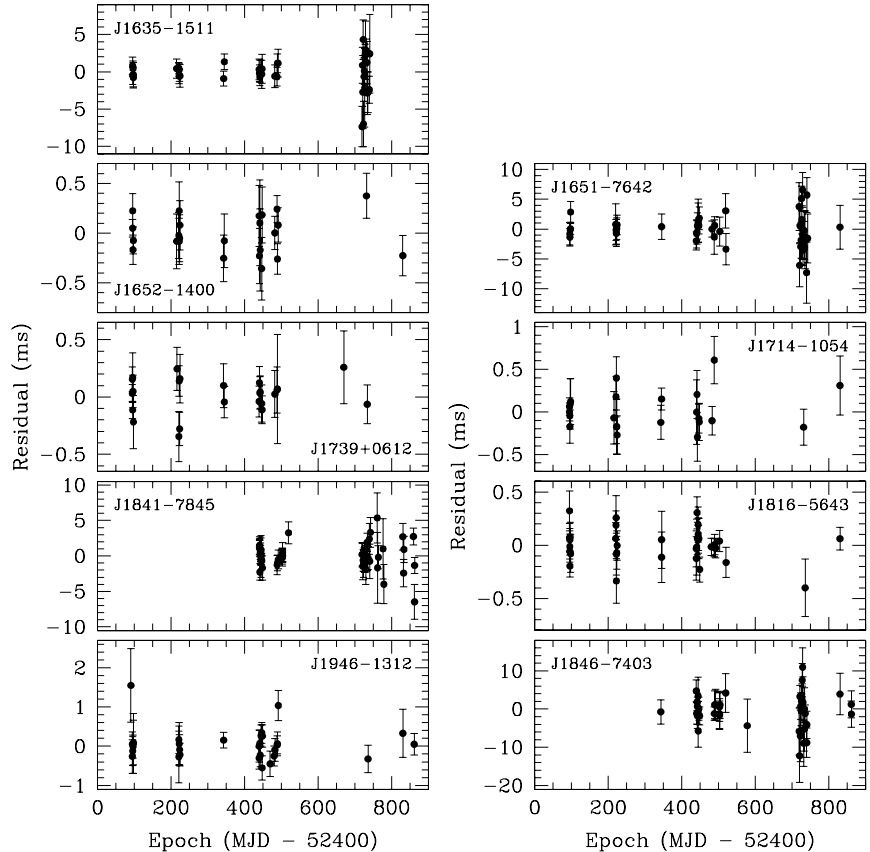

Figure 4. Timing residuals for nine new slow pulsars.

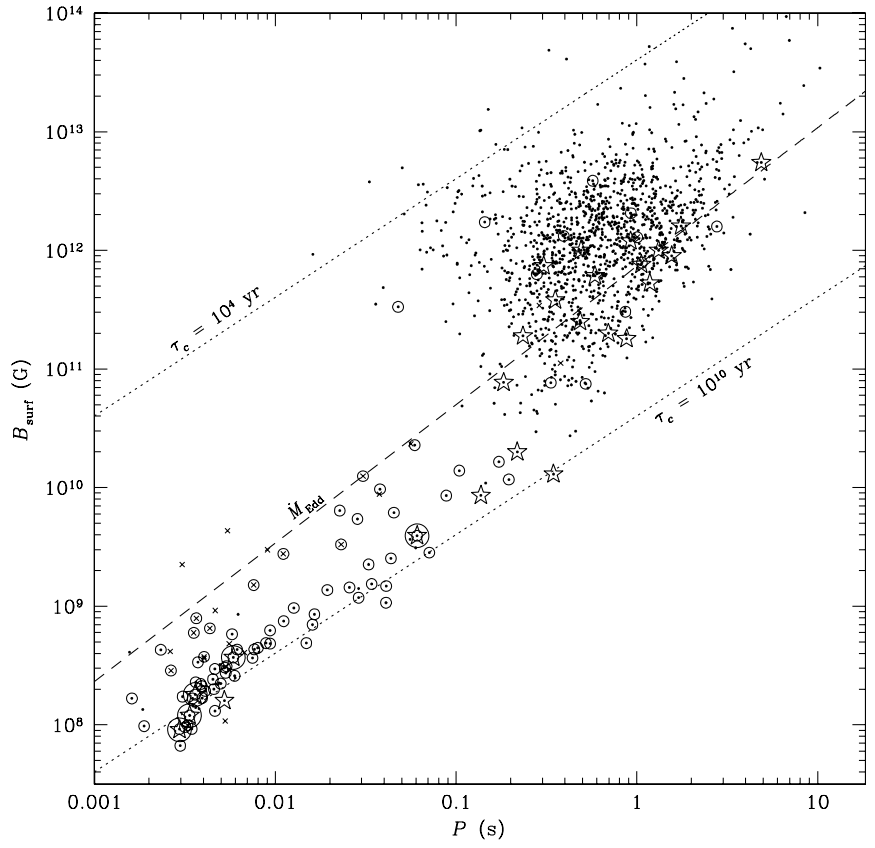

Figure 5. Magnetic field strength - spin period diagram. The dashed line shows the equilibrium spin-up line resulting from Eddington accretion. Dotted lines indicate characteristic ages of $10^{4}$ and $10^{10} \mathrm{yr}$. Small dots mark field objects and crosses designate pulsars in globular clusters; points circumscribed by circles are binary systems. Stars mark pulsars discovered in this survey, except for $\mathrm{J} 1741+1351(P=3.75 \mathrm{~ms})$ for which we do not yet have a secure measurement of $\dot{P}$ and hence $B_{\text {surf }}$. Data are provided by the ATNF Pulsar Catalogue, http://www.atnf.csiro.au/research/pulsar/psrcat/ (Manchester et al. 2005).

original survey integration time before it was convincingly redetected. Thus, the mean flux density of this pulsar was well below our survey limit. This experience highlights the important role played by scintillation in pulsar surveys. Many other candidates which were not as convincing or interesting as this one were, of course, not afforded four confirmation attempts, and there are doubtless other pulsars in the survey region which were not visible at the time of the survey observation. We have 
Table 3

Previously Known Pulsars Detected by Survey

\begin{tabular}{|c|c|c|c|c|c|c|}
\hline \multirow[t]{2}{*}{ Pulsar } & \multicolumn{6}{|c|}{ Parameter $^{\mathrm{a}}$} \\
\hline & $\begin{array}{l}P \\
(\mathrm{~s}) \\
\end{array}$ & $\begin{array}{c}\mathrm{DM} \\
\left(\mathrm{pc} \mathrm{cm}^{-3}\right) \\
\end{array}$ & $\begin{array}{l}S_{1400} \mathrm{~b} \\
(\mathrm{mJy})\end{array}$ & $\begin{array}{c}l \\
(\mathrm{deg})\end{array}$ & $\begin{array}{c}b \\
(\mathrm{deg}) \\
\end{array}$ & $\mathrm{S} / \mathrm{N}^{\mathrm{c}}$ \\
\hline J0711-6830 & 0.0055 & 18.4 & $3.4(5)$ & 279.60 & -23.27 & 14.4 \\
\hline J1034-3224 & 1.1506 & 50.8 & 4.7 & 272.12 & +22.13 & 87.5 \\
\hline B1114-41 & 0.9432 & 40.5 & 3 & 284.52 & +18.08 & 93.1 \\
\hline J1141-3107 & 0.5384 & 30.8 & $\ldots$ & 285.82 & +29.41 & 19.2 \\
\hline $\mathrm{J} 1141-3322$ & 0.2915 & 46.5 & 1 & 286.66 & +27.29 & 76.1 \\
\hline J1159-7910 & 0.5251 & 59.2 & $\ldots$ & 300.48 & -16.54 & 31.4 \\
\hline B1237-41 & 0.5122 & 44.1 & $\ldots$ & 300.76 & +21.42 & 36.0 \\
\hline $\mathrm{J} 1320-3512$ & 0.4585 & 16.4 & $\ldots$ & 309.62 & +27.31 & 63.7 \\
\hline B1325-43 & 0.5327 & 42.0 & 2 & 309.95 & +18.43 & 74.9 \\
\hline J1335-3642 & 0.3992 & 41.7 & $\ldots$ & 312.79 & +25.34 & 11.1 \\
\hline J1418-3921 & 1.0968 & 60.5 & 1.4 & 320.90 & +20.47 & 18.4 \\
\hline B1552-23 & 0.5326 & 51.9 & $0.9(1)$ & 348.52 & +22.50 & 33.5 \\
\hline B1552-31 & 0.5181 & 73.0 & $1.4(4)$ & 342.78 & +16.76 & 64.6 \\
\hline B1600-27 & 0.7783 & 46.2 & $1.7(3)$ & 347.20 & +18.77 & 76.9 \\
\hline J1603-2531 & 0.2831 & 53.8 & $\ldots$ & 348.45 & +19.99 & 225.2 \\
\hline B1620-09 & 1.2764 & 68.2 & $0.6(1)$ & 5.38 & +27.18 & 34.4 \\
\hline $\mathrm{B} 1620-26^{\mathrm{d}}$ & 0.0111 & 62.9 & $2.0(3)$ & 351.05 & +15.96 & 22.2 \\
\hline J1643-1224 & 0.0046 & 62.4 & $3.3(1)$ & 5.75 & +21.22 & 62.9 \\
\hline B1641-68 & 1.7856 & 43.0 & 2 & 321.91 & -14.82 & 28.1 \\
\hline B1642-03 & 0.3877 & 35.7 & $21.0(6)$ & 14.19 & +26.06 & 443.3 \\
\hline B1701-75 & 1.1910 & 37.0 & $\ldots$ & 316.75 & -20.21 & 29.9 \\
\hline $\mathrm{J} 1713+0747^{\mathrm{e}}$ & 0.0046 & 16.0 & 3 & 28.83 & +25.21 & 10.1 \\
\hline B1718-02 & 0.4777 & 67.0 & $1.0(2)$ & 20.21 & +18.93 & 15.6 \\
\hline B1726-00 & 0.3860 & 41.1 & $\ldots$ & 23.11 & +18.28 & 20.1 \\
\hline $\mathrm{J} 1736+05$ & 0.9992 & 42.0 & $\ldots$ & 29.67 & +19.20 & 205.0 \\
\hline B $1737+13$ & 0.8031 & 48.7 & $3.9(5)$ & 37.16 & +21.67 & 86.8 \\
\hline $\mathrm{J} 1740+1000$ & 0.1541 & 23.9 & $9.2(4)$ & 34.09 & +20.26 & 11.6 \\
\hline B1806-53 & 0.2610 & 45.0 & $\ldots$ & 340.36 & -15.90 & 97.4 \\
\hline B1828-60 & 1.8894 & 35.0 & $\ldots$ & 334.89 & -21.19 & 36.3 \\
\hline J1932-3655 & 0.5714 & 59.9 & $\ldots$ & 2.14 & -23.55 & 31.2 \\
\hline B1937-26 & 0.4029 & 50.0 & $3(1)$ & 13.97 & -21.83 & 40.3 \\
\hline B1940-12 & 0.9724 & 28.9 & $0.7(2)$ & 27.32 & -17.16 & 31.1 \\
\hline B1941-17 & 0.8412 & 56.3 & 0.2 & 22.38 & -19.43 & 15.1 \\
\hline B1943-29 & 0.9594 & 44.3 & $0.8(3)$ & 11.17 & -24.12 & 20.6 \\
\hline B2003-08 & 0.5809 & 32.4 & $2.8(7)$ & 34.17 & -20.31 & 122.7 \\
\hline В2043-04 & 1.5469 & 35.8 & $1.7(5)$ & 42.74 & -27.40 & 50.6 \\
\hline
\end{tabular}

\section{Notes.}

${ }^{a}$ Parameter values except for detected S/N obtained from http://www.atnf.csiro.au/research/pulsar/psrcat/.

${ }^{\mathrm{b}}$ Figures in parentheses are uncertainty in the last digit quoted, where known.

${ }^{\mathrm{c}}$ For pulsars detected in multiple survey beams, $\mathrm{S} / \mathrm{N}$ of strongest detection.

${ }^{\mathrm{d}}$ In the globular cluster M4.

${ }^{\mathrm{e}}$ Discovered at subharmonic of actual spin frequency.

no doubt that reobserving regions which have previously been surveyed will yield new discoveries, even when a new observing capability or strategy is not brought to bear.

The fraction of binary and recycled pulsars detected in this survey ( 7 out of 62 pulsars) is nearly twice that of the pulsar population as a whole (excluding pulsars in globular clusters), demonstrating the efficacy of a shallow, high-frequency survey away from the Galactic plane for finding MSPs. Figure 6 shows the period distribution of newly discovered pulsars with previously known pulsars in the survey region, excluding those in globular clusters. The distribution of new pulsar periods is clearly weighted toward short periods relative to the previously known pulsars even though MSPs are thought to have slightly steeper spectra than slow pulsars (Lorimer et al. 1995; Toscano et al. 1998), suggesting that the improved effective time resolution afforded by observing at high frequency is a significant advantage compared with the sensitivity of the Parkes
Southern Pulsar Survey at $436 \mathrm{MHz}$ (Manchester et al. 1996). The DM distribution of newly discovered pulsars is similar to that of the previously known pulsars (Figure 7). Figure 8 shows the galactic distribution of newly discovered pulsars. It is curious that while most of the new pulsars are in the northern side of the galaxy, the distribution of new pulsars in the south is relatively isotropic in the survey region, while in the north, it falls off markedly with increasing distance from the Galactic plane.

The timing program described above also included a number of previously unsolved slow pulsars discovered in the Swinburne Intermediate Latitude Pulsar Survey. Timing model parameters for nine of these pulsars are given in Table 5 .

\section{IMPLICATIONS FOR SUBMILLISECOND PULSARS}

It is curious that the first millisecond pulsar discovered, PSR B1937+21 with $P=1.56 \mathrm{~ms}$ (Backer et al. 1982), remained the 
Table 4

Undetected Previously Known Pulsars in Survey Region

\begin{tabular}{|c|c|c|c|c|c|}
\hline \multirow[t]{2}{*}{ Pulsar } & \multicolumn{5}{|c|}{ Parameter $^{\mathrm{a}}$} \\
\hline & $\begin{array}{l}P \\
\text { (s) }\end{array}$ & $\begin{array}{c}\mathrm{DM} \\
\left(\mathrm{pc} \mathrm{cm}{ }^{-3}\right)\end{array}$ & $\begin{array}{l}S_{1400^{\mathrm{b}}} \\
(\mathrm{mJy})\end{array}$ & $\begin{array}{c}l \\
\text { (deg) }\end{array}$ & $\begin{array}{c}b \\
\text { (deg) }\end{array}$ \\
\hline B0559-57 & 2.2614 & 30.0 & $\ldots$ & 266.56 & -29.33 \\
\hline B0904-74 & 0.5496 & 51.1 & $\ldots$ & 289.81 & -18.31 \\
\hline B0909-71 & 1.3629 & 54.3 & $\ldots$ & 287.80 & -16.24 \\
\hline B1010-23 & 2.5179 & 22.5 & $\ldots$ & 262.20 & +26.39 \\
\hline $\mathrm{J} 1047-3032$ & 0.3303 & 52.5 & 0.18 & 273.57 & +25.14 \\
\hline B1056-78 & 1.3474 & 51.0 & $\ldots$ & 297.64 & -17.56 \\
\hline B1118-79 & 2.2806 & 27.4 & $\ldots$ & 298.77 & -17.48 \\
\hline $\mathrm{J} 1455-3330$ & 0.0080 & 13.6 & $1.2(1)$ & 330.80 & +22.57 \\
\hline B1607-13 & 1.0184 & 49.1 & $\ldots$ & 359.51 & +26.95 \\
\hline B1612-29 & 2.4776 & 44.8 & $\ldots$ & 347.46 & +15.06 \\
\hline $\mathrm{J} 1632-1013$ & 0.7176 & 89.9 & $\ldots$ & 5.91 & +24.64 \\
\hline $\mathrm{J} 1650-1654$ & 1.7496 & 43.2 & 1.6 & 2.93 & +17.23 \\
\hline B1648-17 & 0.9734 & 33.5 & $0.3(1)$ & 2.89 & +16.88 \\
\hline B1657-13 & 0.6410 & 60.4 & $\ldots$ & 7.58 & +17.59 \\
\hline $\mathrm{J} 1720+2150$ & 1.6157 & 41.1 & $\ldots$ & 44.03 & +29.36 \\
\hline $\mathrm{J} 1725-0732$ & 0.2399 & 58.9 & $\ldots$ & 15.87 & +15.32 \\
\hline B1732-02 & 0.8394 & 65.0 & $\ldots$ & 21.98 & +15.92 \\
\hline $\mathrm{J} 1752+2359$ & 0.4091 & 36.0 & $\ldots$ & 49.17 & +23.06 \\
\hline $\mathrm{J} 1756+18$ & 0.7440 & 77.0 & $\ldots$ & 43.82 & +20.21 \\
\hline J1813+1822 & 0.3364 & 60.8 & $\ldots$ & 45.62 & +16.39 \\
\hline B1851-79 & 1.2792 & 39.0 & $\ldots$ & 314.39 & -27.05 \\
\hline $\mathrm{J} 1910-5959 \mathrm{~A}^{\mathrm{c}}$ & 0.0033 & 33.7 & 0.22 & 336.59 & -25.73 \\
\hline $\mathrm{J} 1910-5959 \mathrm{~B}^{\mathrm{c}}$ & 0.0084 & 33.3 & 0.06 & 336.56 & -25.62 \\
\hline $\mathrm{J} 1910-5959 \mathrm{C}^{\mathrm{c}}$ & 0.0053 & 33.2 & 0.30 & 336.53 & -25.66 \\
\hline $\mathrm{J} 1910-5959 \mathrm{D}^{\mathrm{c}}$ & 0.0090 & 33.3 & 0.07 & 336.56 & -25.62 \\
\hline $\mathrm{J} 1910-5959 \mathrm{E}^{\mathrm{c}}$ & 0.0046 & 33.3 & 0.09 & 336.56 & -25.62 \\
\hline $\mathrm{J} 1940-2403$ & 1.8553 & 63.3 & $\ldots$ & 15.91 & -21.01 \\
\hline J1947-4215 & 1.7981 & 35.0 & $\ldots$ & 357.25 & -27.71 \\
\hline B1946-25 & 0.9576 & 23.1 & $0.4(1)$ & 15.33 & -23.39 \\
\hline J2005-0020 & 2.2797 & 35.9 & 0.4 & 41.40 & -16.64 \\
\hline
\end{tabular}

Notes.

a Parameter values obtained from http://www.atnf.csiro.au/research/pulsar/ psrcat/.

${ }^{\mathrm{b}}$ Figures in parenthesis are uncertainty in the last digit quoted, where known.

${ }^{\mathrm{c}}$ In the globular cluster NGC 6752.

fastest known for over two decades. Like the recently discovered record holder, PSR J1748-2446AD with $P=1.40 \mathrm{~ms}$ (Hessels et al. 2006), it was discovered in a targeted search. Until fairly recently, few large-area pulsar surveys such as the one described here were sensitive to pulsars with spin periods of order one millisecond. It is interesting to ask whether the lack of observed pulsars with periods faster than that of J1748-2446AD can be explained by observational selection effects, or if it requires these putative "submillisecond" pulsars to be an extremely rare population.

The temporal smearing due to dispersion $\tau_{\mathrm{DM}}$ as a function of DM in a given pulsar survey is dictated by both the observed frequency $v$ (in $\mathrm{GHz}$ ) and the channel bandwidth $B(\mathrm{MHz})$ according to

$$
\tau_{\mathrm{DM}}=8.3 \mu \mathrm{s} \frac{B \mathrm{DM}}{\nu^{3}},
$$

where DM is the dispersion measure in the usual units of $\mathrm{pc} \mathrm{cm}^{-3}$. For all of the Parkes multibeam surveys, this relation leads to a smearing of $1 \mathrm{~ms}$ at $\mathrm{DM}=100 \mathrm{pc} \mathrm{cm}^{-3}$ rendering true submillisecond pulsars invisible at moderate to large values of DM. As can be seen from Figure 7, there was only one pulsar detected beyond a DM of $100 \mathrm{pc} \mathrm{cm}^{-3}$ in this survey, which may indicate that the maximum DM of Galactic pulsars

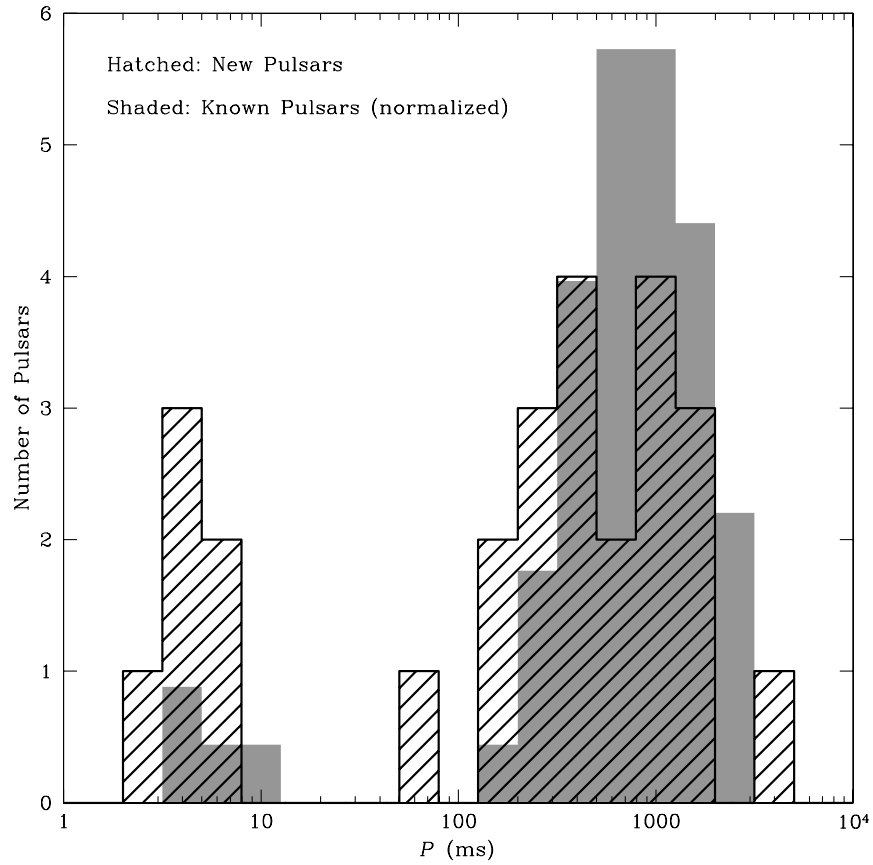

Figure 6. Spin period distribution of pulsars in survey region. The hatched histogram shows the distribution of the 26 newly discovered pulsars, while the gray-shaded histogram shows the 59 previously known field pulsars in the survey region, normalized to have the same total area as the new pulsars.

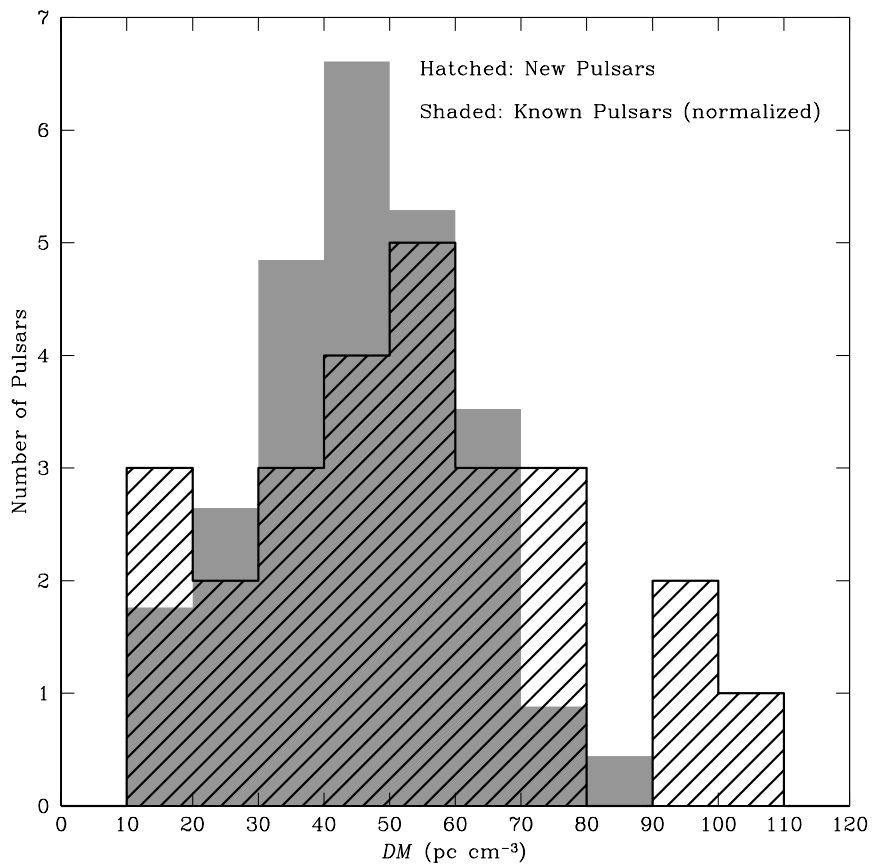

Figure 7. Dispersion measure distribution of pulsars in survey region. The hatched histogram shows the distribution of the 26 newly discovered pulsars, while the gray-shaded histogram shows the 59 previously known field pulsars in the survey region, normalized to have the same total area as the new pulsars.

in the survey volume is close to that required to render a submillisecond pulsar invisible. Although it is possible to model a putative population of submillisecond pulsars based upon what we know about millisecond pulsars and place some limits on their numbers, it is difficult to model human interaction with real pulsar candidates. A pulsar candidate is always more convincing when several harmonics are detected, making it easier to discriminate against the literally thousands of 
Table 5

Timing Model Parameters for Nine Slow Pulsars from Swinburne Intermediate Latitude Pulsar Survey

\begin{tabular}{|c|c|c|c|c|c|c|}
\hline \multirow[t]{2}{*}{ Pulsar } & \multicolumn{6}{|c|}{ Parameter $^{\mathrm{a}}$} \\
\hline & $\alpha(\mathrm{J} 2000)$ & $\delta(\mathrm{J} 2000)$ & $\begin{array}{l}P \\
(\mathrm{~s})\end{array}$ & $\begin{array}{c}P \text { Epoch } \\
\text { (MJD) }\end{array}$ & $\begin{array}{c}\dot{P} \\
\left(10^{-15}\right)\end{array}$ & $\begin{array}{c}D M^{\mathrm{b}} \\
\left(\mathrm{pc} \mathrm{cm}^{-3}\right)\end{array}$ \\
\hline J1057-4754 & $10^{\mathrm{h}} 57^{\mathrm{m}} 53^{\mathrm{s}} .138(7)$ & $-47^{\circ} 54^{\prime} 57^{\prime \prime} 60(9)$ & $0.628305878552(7)$ & 53000.0 & $0.2248(2)$ & 60 \\
\hline $\mathrm{J} 1232-4742$ & $12^{\mathrm{h}} 32^{\mathrm{m}} 19^{\mathrm{s}} 1(2)$ & $-47^{\circ} 42^{\prime} 50^{\prime} 9(15)$ & $1.8729943770(2)$ & 53000.0 & $0.014(5)$ & $26(3)$ \\
\hline $\mathrm{J} 1423-6953$ & $14^{\mathrm{h}} 23^{\mathrm{m}} 26^{\mathrm{s}} .79(2)$ & $-69^{\circ} 53^{\prime} 42^{\prime \prime} 82(8)$ & $0.333410710172(3)$ & 53000.0 & $1.45130(6)$ & $123.98(12)$ \\
\hline J1535-4415 & $15^{\mathrm{h}} 35^{\mathrm{m}} 55^{\mathrm{s}} .92(7)$ & $-44^{\circ} 15^{\prime} 08^{\prime \prime} 4(12)$ & $0.46840160422(3)$ & 53000.0 & $0.0405(9)$ & $110.7(5)$ \\
\hline J1537-4912 & $15^{\mathrm{h}} 37^{\mathrm{m}} 28^{\mathrm{s}} \cdot 2(2)$ & $-49^{\circ} 12^{\prime} 03^{\prime \prime}(3)$ & $0.30131077523(8)$ & 53000.0 & $1.932(2)$ & $69.7(14)$ \\
\hline J1539-6322 & $15^{\mathrm{h}} 39^{\mathrm{m}} 24^{\mathrm{s}} .70(3)$ & $-63^{\circ} 22^{\prime} 53^{\prime \prime} .2(2)$ & $1.63084630957(3)$ & 53000.0 & $0.2016(6)$ & $163.5(3)$ \\
\hline J1706-6118 & $17^{\mathrm{h}} 06^{\mathrm{m}} 09^{\mathrm{s}} .794(6)$ & $-61^{\circ} 18^{\prime} 11^{\prime \prime} .70(10)$ & $0.3619213249276(15)$ & 53000.0 & $0.29213(4)$ & $76.13(6)$ \\
\hline $\mathrm{J} 1806+1023$ & $18^{\mathrm{h}} 06^{\mathrm{m}} 52^{\mathrm{s}} .114(3)$ & $+10^{\circ} 23^{\prime} 18^{\prime \prime} .30(12)$ & $0.484286459778(2)$ & 53000.0 & $0.05725(4)$ & $52.03(7)$ \\
\hline J1824-2537 & $18^{\mathrm{h}} 24^{\mathrm{m}} 30^{\mathrm{s}} \cdot 587(10)$ & $-25^{\circ} 37^{\prime} 19^{\prime \prime}(2)$ & $0.223320267185(3)$ & 53000.0 & $0.44016(8)$ & $158.5(2)$ \\
\hline
\end{tabular}

Notes.

${ }^{\text {a }}$ Figures in parenthesis are uncertainties in the last digit quoted. Uncertainties are calculated from twice the formal error produced by TEMPO.

b Where no uncertainty is indicated, DM was taken from Edwards et al. (2001).

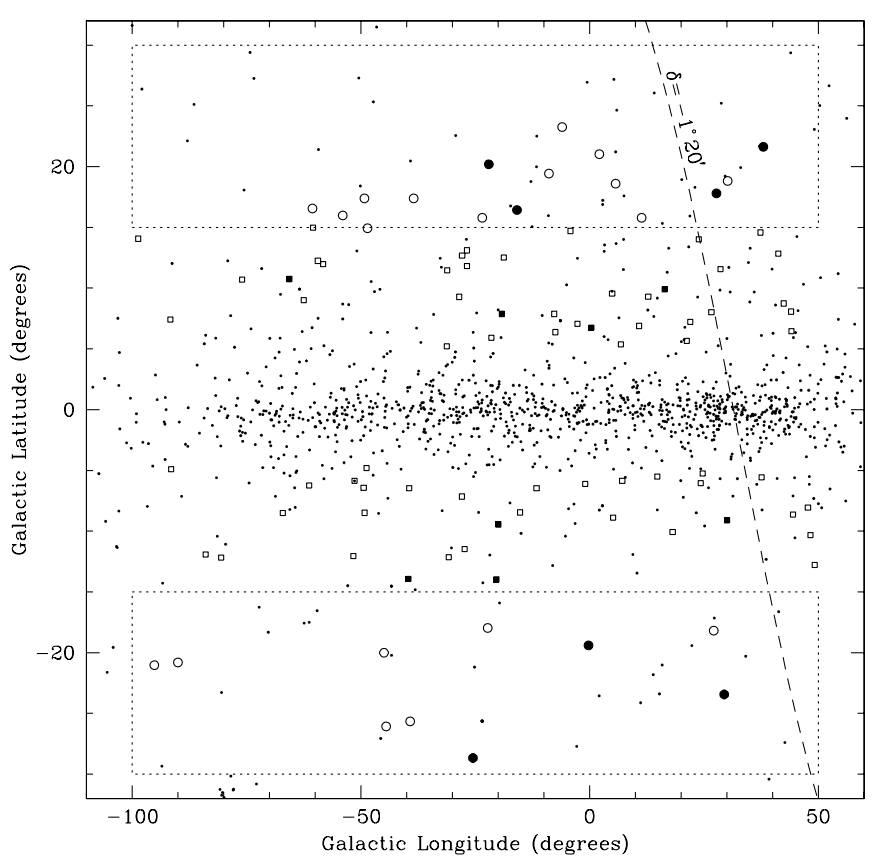

Figure 8. Galactic distribution of pulsars. The survey region is denoted by the dashed boxes, and newly discovered pulsars are shown as circles in these regions. Open circles represent the 19 slow pulsars reported here, while filled circles denote the seven new recycled pulsars. Open and filled squares, respectively, represent the slow and recycled pulsars discovered in the previous Swinburne Intermediate Latitude Pulsar Survey, and other known pulsars are shown as points. The diagonal dashed curve represents the southern declination limit of the Arecibo telescope; the area to the right of the curve is visible to Arecibo.

sinusoidal candidates generated by RFI. Definitive statements about the submillisecond pulsar population will require surveys with very high time and frequency resolution, such as the PALFA survey at Arecibo (Cordes et al. 2006). For now, we simply state that submillisecond pulsars appear to be at best relatively rare compared to the millisecond pulsar population.

\section{CONCLUSIONS}

We have completed a survey for pulsars covering $10 \%$ of the sky with the $21 \mathrm{~cm}$ multibeam receiver system at the $64 \mathrm{~m}$ Parkes radio telescope. This survey was very successful, discovering seven new recycled pulsars and 19 slow pulsars, and redetecting 36 previously known pulsars in the survey region.

Taken together with the previous Swinburne Intermediate Latitude Survey, which discovered 69 new pulsars including 8 recycled pulsars, we now have a consistent census of the pulsar population over $\sim 7100$ square degrees between $5^{\circ}$ and $30^{\circ}$ from the Galactic plane, which will be very valuable for modeling the underlying population.

The four major pulsar surveys using the Parkes multibeam receiver and associated filterbanks (this work, the Swinburne Intermediate Latitude Pulsar Survey, the Parkes Multibeam Pulsar Survey, and the Parkes High Latitude Pulsar Survey) have more than doubled the total number of pulsars known, and have led to the discovery of objects that span a broad range of parameter space: high-magnetic field pulsars (Camilo et al. 2000), young pulsars (Kramer et al. 2003), binaries with massive companions (Stairs et al. 2001), intermediate mass binary pulsars with massive white dwarf companions (Camilo et al. 2001), the double pulsar PSR J0737-3039A/B (Lyne et al. 2004), and high timing precision millisecond pulsars such as PSR J1909-3744 (Jacoby et al. 2005). Pulsar surveys make breakthroughs when they cover a significant region of parameter space that was previously unsearched. The Parkes multibeam surveys covered a large area at a new frequency with good sensitivity to achieve impressive results. To break new ground, new surveys will have to improve their sensitivity through higher time and frequency resolution, or the use of larger telescopes. New field-programmable gate arrays permit the construction of relatively inexpensive digital filterbanks that can deliver very fine frequency and time resolution with multibit sampling. An obvious follow-up to the Parkes multibeam surveys would be to build a digital filterbank with ten times finer frequency resolution, allowing surveys to penetrate larger column densities of electrons.

We thank H. Knight, A. Hotan, and W. van Straten for help with survey observations, and the Parkes Multibeam Pulsar Survey collaboration for making the data acquisition hardware and software used for this survey available to the community. The Parkes telescope is part of the Australia Telescope, which is funded by the Commonwealth of Australia for operation as a National Facility managed by CSIRO. B.A.J. and S.R.K. thank NSF and NASA for supporting their research. B.A.J. held a 
National Research Council Research Associateship Award at the Naval Research Laboratory (NRL) during part of this work. Basic research in radio astronomy at NRL is supported by the Office of Naval Research.

\section{REFERENCES}

Backer, D. C., Kulkarni, S. R., Heiles, C., Davis, M. M., \& Goss, W. M. 1982, Nature, 300, 615

Burgay, M., et al. 2006, MNRAS, 368, 283

Camilo, F., Kaspi, V. M., Lyne, A. G., Manchester, R. N., Bell, J. F., D’Amico, N., McKay, N. P. F., \& Crawford, F. 2000, ApJ, 541, 367

Camilo, F., et al. 2001, ApJ, 548, L187

Cordes, J. M., \& Lazio, T. J. W. 2002, arXiv:astro-ph/0207156

Cordes, J. M., et al. 2006, ApJ, 637, 446

Edwards, R. T., \& Bailes, M. 2001, ApJ, 553, 801

Edwards, R. T., Bailes, M., van Straten, W., \& Britton, M. C. 2001, MNRAS, 326,358

Hessels, J. W. T., Ransom, S. M., Stairs, I. H., Freire, P. C. C., Kaspi, V. M., \& Camilo, F. 2006, Science, 311, 1901
Hewish, A., Bell, S. J., Pilkington, J. D. H., Scott, P. F., \& Collins, R. A. 1968, Nature, 217, 709

Jacoby, B. A. 2005, PhD thesis, California Inst. Technol.

Jacoby, B. A., Bailes, M., Ord, S. M., Knight, H. S., \& Hotan, A. W. 2007, ApJ, 656,408

Jacoby, B. A., Bailes, M., van Kerkwijk, M. H., Ord, S., Hotan, A., Kulkarni, S. R., \& Anderson, S. B. 2003, ApJ, 599, L99

Jacoby, B. A., Hotan, A., Bailes, M., Ord, S., \& Kulkarni, S. R. 2005, ApJ, 629, L113

Kramer, M., et al. 2003, MNRAS, 342, 1299

Lorimer, D. R., Yates, J. A., Lyne, A. G., \& Gould, D. M. 1995, MNRAS, 273, 411

Lorimer, D. R., et al. 2006, MNRAS, 372, 777

Lyne, A. G., et al. 2004, Science, 303, 1153

Manchester, R. N., Hobbs, G. B., Teoh, A., \& Hobbs, M. 2005, AJ, 129, 1993

Manchester, R. N., et al. 1996, MNRAS, 279, 1235

Manchester, R. N., et al. 2001, MNRAS, 328, 17

Stairs, I. H., et al. 2001, MNRAS, 325, 979

Staveley-Smith, L., et al. 1996, PASA, 13, 243

Taylor, J. H. 1974, A\&AS, 15, 367

Toscano, M., Bailes, M., Manchester, R., \& Sandhu, J. 1998, ApJ, 506, 863 